This is a post-peer-review, pre-copyedit version of an article published in Photonic Network Communications. The final authenticated version is available online at:

https://doi.org/10.1007/s11107-020-00903-x

\title{
Spatial Partitioning for Proactive Spectrum Fragmentation Avoidance in Flex-Grid/SDM Dynamic Optical Core Networks
}

\author{
Jaume Comellas, Jordi Perelló, Josep Solé-Pareta, Gabriel Junyent\#
}

Advanced Broadband Communications Center (CCABA), Universitat Politècnica de Catalunya (UPC), Jordi Girona 1-3, 08034 Barcelona Spain, e-mail: comellas@tsc.upc.edu

\begin{abstract}
Spectrum fragmentation has always been a major issue to overcome toward spectrally efficient Flex-Grid over Single-Mode Fiber (SMF) dynamic optical networks, and continues like so when evolving to Flex-Grid over Spatial Division Multiplexing (SDM). A possible strategy to eliminate its pernicious effects is to divide the available spectrum into several partitions, dedicating each one of them to only support connections with identical spectral requirements. In this way, a first-fit spectrum assignment ensures that spectral gaps at each spectral partition will always match the bandwidth needs of future connection requests. In this paper, we extend this strategy to be applicable to Flex-Grid/SDM dynamic optical networks. Furthermore, leveraging the spatial multiplicity offered by SDM, we also investigate spatial partitioning as an effective yet simpler and more easily manageable solution to also eliminate the spectrum fragmentation negative effects. Both strategies are numerically evaluated in two reference Flex-Grid/SDM backbone networks with $x 7$ spatial multiplicity, yielding noteworthy carried network load gains up to $18 \%$ versus a non-partitioned network scenario. When increasing the spatial multiplicities up to $\times 30$, such gains tend to stabilize around 3-4\%. Some results are also obtained under unexpected traffic profile deviations, showing that, even under moderate deviations, partitioning becomes beneficial. Comparing spectral and spatial partitioning, they tend to perform quite similar in all cases. This makes us advocate for spatial partitioning as a more interesting solution for spectrum fragmentation avoidance in this kind of networks.
\end{abstract}

\section{INTRODUCTION}

Elastic Optical Networks (EONs) implementing Flex-Grid technology [1][2] have become top candidates to realize the upcoming data transport network infrastructures, given their superior spectral efficiency versus legacy Wavelength Division Multiplexing (WDM)-based ones [3]. In Flex-Grid, the optical fiber spectrum is discretized into Frequency Slots (FSs) of $12.5 \mathrm{GHz}$ width, following ITU-T Recommendation G.694.1 [4], which can be contiguously reserved to form channels tailored to any signal bandwidth. This enables the efficient allocation of subwavelength and ultra-high bit-rate connections together onto the same optical network infrastructure, being the latter also called super-channels, composed of multiple adjacent subchannels over separate optical carriers.

However, recent studies have revealed that even adopting Flex-Grid, the capacity required to 
support mid- and long-term traffic forecasts can go beyond the nonlinear Shannon limit of standard Single-Mode Fibers (SMFs), incurring a potential "capacity crunch" [5]. To avoid this situation, the introduction of Spatial Division Multiplexing (SDM) into the optical layer seems mandatory [6]. With this objective, Single Mode Fiber Bundles (SMFBs) can be equipped per link in a near-term, thus multiplying the Flex-Grid network capacity by the number of physically independent SMFs per bundle. The mid- and long-term fully-SDM vision is expected to rely on advanced optical fiber technologies [7], like Multi-Core Fibers (MCFs), Few-Mode Fibers (FMFs) and even Few-Mode MCFs (FM-MCFs), which should foster integrated system components like transponders, amplifiers or Re-configurable Optical Add \& Drop Multiplexers (ROADMs).

With Flex-Grid/SDM coming to the fore, new super-channel allocation options appear [8]. These ones range from spectral super-channels, as in Flex-Grid over SMF networks, where their composing sub-channels are all arranged across the spectral domain, to spatial ones with their sub-channels arranged across the spatial domain (over the same central frequency). Mixed options (i.e., spectral-spatial super-channels) may also be possible, arranging their sub-channels across both spectral and spatial domains. Among these options, spatial super-channels are considered the most cost-effective, as a simpler SDM-ROADM architecture realizing Joint Switching (JoS) can be employed [9]. Spectral and spatial-spectral super-channel options should yield higher spectral efficiency than the spatial ones but requiring, in principle, expensive SDMROADM architectures able to switch any spectral portion from any input fiber/core/mode to any output one [10], a technique called Independent Switching (InS). An alternative cheaper SDMROADM architecture still enabling spectral and spectral-spatial super-channels is the one performing InS without lane change [11], which trades fiber/core/mode continuity for a simpler SDM-ROADM architecture, comparable to that realizing JoS. Besides, InS without lane change offers a spectral efficiency close to that obtained with pure InS [12].

From the very beginning, spectrum fragmentation has been identified as a major issue to address for delivering high spectrum efficiency and utilization in EONs [2]. It results from the allocation/release of spectral resources to connections with different bandwidth requirements upon their arrival/departure, leaving spectral gaps of different widths that complicate the allocation of future connections, particularly those with high spectral requirements.

To mitigate the spectrum fragmentation negative effects, proactive and reactive solutions have been proposed in the literature [13]. Reactive solutions aim at defragmenting the optical fiber spectrum during network operation, re-arranging active connections in a way that the fragmentation of the spectrum is reduced, thus facilitating the potential allocation of future connection requests. During this operation, it is of utmost importance not to disrupt active connections carrying end-user traffic that is, performing a "hitless" defragmentation. Conversely, proactive solutions seek to allocate new connections trying to minimize the resulting fragmentation after their allocation (e.g., as in [14][15]). It is noteworthy that spectrum fragmentation is not only present in pure EONs but also in Flex-Grid/SDM networks, for which reactive and proactive solutions to mitigate its effects have also been recently proposed in the literature (e.g., see [16][17]).

A proactive solution proposed in the literature to avoid the pernicious effects of the spectrum 
fragmentation in Flex-Grid optical networks consists in dividing the spectral resources, so that each of the resulting partitions is dedicated to only support connections with identical bandwidth requirements [18][19]. In this way, by applying a simple first-fit spectrum assignment strategy, the width of the available spectrum gaps in each partition will always match the bandwidth requirements of future connections to be allocated there. A critical aspect to achieve high spectral utilization with this solution is to assign an appropriate amount of spectrum to each partition, for example, based on expected load and traffic profile.

The present paper has a three-fold objective. First of all, we analyze the benefits and drawbacks of extending the aforementioned proactive spectrum fragmentation avoidance solution (hereafter generically referred to as spectral partitioning) to Flex-Grid/SDM networks, where spectrum fragmentation still exists and needs to be addressed as well. Secondly, taking advantage of the spatial multiplicity offered by SDM, we propose an alternative proactive spectrum fragmentation solution relying on spatial partitioning, which aims at achieving identical Flex-Grid/SDM network performance as the previous spectrum partitioning solution, but being simpler and more easily manageable. Thirdly, we numerically compare the performance of both solutions in two Flex-Grid/SDM reference backbone network scenarios with spatial multiplicities up to $\times 30$, so as to extract our final conclusions.

At this point, we should emphasize that this paper focuses on short-term realizable FlexGrid/SDM networks equipping bundles of independent SMFs per link, which do not suffer from crosstalk-related impairments. However, crosstalk can play a critical role on the maximum transmission reach of optical signals with advanced SDM technologies like MCFs, FMFs or FMMCFs, envisioned in mid- and long-term Flex-Grid/SDM network realizations. Hence, although not considered in this work hereafter, crosstalk would require special attention when extending our proposed solutions to networks using such advanced SDM technologies, demanding the use of crosstalk-aware transmission reach estimation models, as the ones presented in [20].

The remainder of this paper continues as follows. Section 2 presents the extension of the spectral partitioning solution to Flex-Grid/SDM networks, as well as the newly proposed spatial partitioning solution. Section 3 presents the evaluation scenarios, obtained numerical results and eventual comparison. Finally, section 4 draws up the main conclusions of this work.

\section{PROACTIVE FRAGMENTATION AVOIDANCE}

A common characteristic of any Flex-Grid/SDM scenario is the coexistence of heterogeneous connections, such that the allocated resources for them adapt to their bandwidth requirements. This is seen as a major advantage in terms of network use effectiveness, largely demonstrated when having to transport any given traffic. Many previous works dealt with multi-rate connections, whose spectral needs vary depending on the modulation formats allowed. In general, different bit-rate connections are generated and coexist during network operation. Considering the available technology, many works currently focus on dynamic network scenarios supporting 100G, 400G and $1 \mathrm{~Tb} / \mathrm{s}$ connections, tailoring the spectrum assigned to them according to their capacity, which produces spectral fragmentation.

Extending the idea provided in [14] and [15] for Flex-Grid/SMF networks to Flex-Grid/SDM ones, 
we propose to isolate the different connection types by assigning different spectral resource partitions dedicated to each one of them, what we refer to as spectral partitioning. According to the traffic profile offered to the network, different spectrum partitions are created, whose size depends on the specific offered traffic contribution by each type of connections. These partitions can include spectral resources of multiple spatial channels present in the FlexGrid/SDM network links.

In order to describe how spectral partitioning can be performed in a Flex-Grid/SDM network, imagine a network traffic profile composed of $N$ traffic types (i.e., bit-rates), $T_{1}, T_{2}, \ldots, T_{N}$, with respective probabilities $p_{1}, p_{2}, \ldots, p_{N}$, being $p_{n}$ probability that an incoming demand is of type $T_{n}$, $n \in\{1, \ldots, N\}$.

Imagine now for simplicity that fixed baud-rate modulators are used in the network and a single modulation format is always employed. Under these assumptions, the effective bit-rate per Optical Carrier (OC), denoted as $C$, can be obtained by multiplying the fixed baud-rate of the modulators (in Gbaud) and the efficiency of the modulation format used (in b/s/Hz). In this way, the number of OCs needed to configure a super-channel (a spectral super-channel in this work) able to support a demand of type $T_{n}$, denoted as $N O C_{n}$, would be $N O C_{n}=$ ceil $\left(T_{n} / C\right)$, being ceil() the ceiling function. Finally, having $N O C_{n}$ values for all $n \in\{1, \ldots, N\}$, we can now proceed to obtain the relative spectrum requirements per traffic type, denoted as $R_{1}, R_{2}, \ldots, R_{N}$, so that $R_{n}=$ $p_{n} \cdot N O C_{n} /\left(p_{1} \cdot N O C_{1}+p_{2} \cdot N O C_{2}+\ldots+p_{N} \cdot N O C_{N}\right)$, for all $n \in\{1, \ldots, N\}$.

With these relative spectrum requirements per traffic type, spectral partitioning can be performed in a Flex-Grid/SDM network as follows. Firstly, the total number of frequency slots

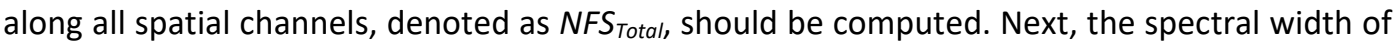
each spectral partition dedicated to each traffic type, denoted as $N F S_{n}$, for all $n \in\{1, \ldots, N\}$, should also have to be computed as $N F S_{n}=$ round $\left(R_{n} \cdot N F S_{\text {Total }}\right.$ ), being round() an integer rounding function. Finally, spectral partitions of such computed widths would be configured. This procedure is depicted in the flowchart presented in Figure 1.

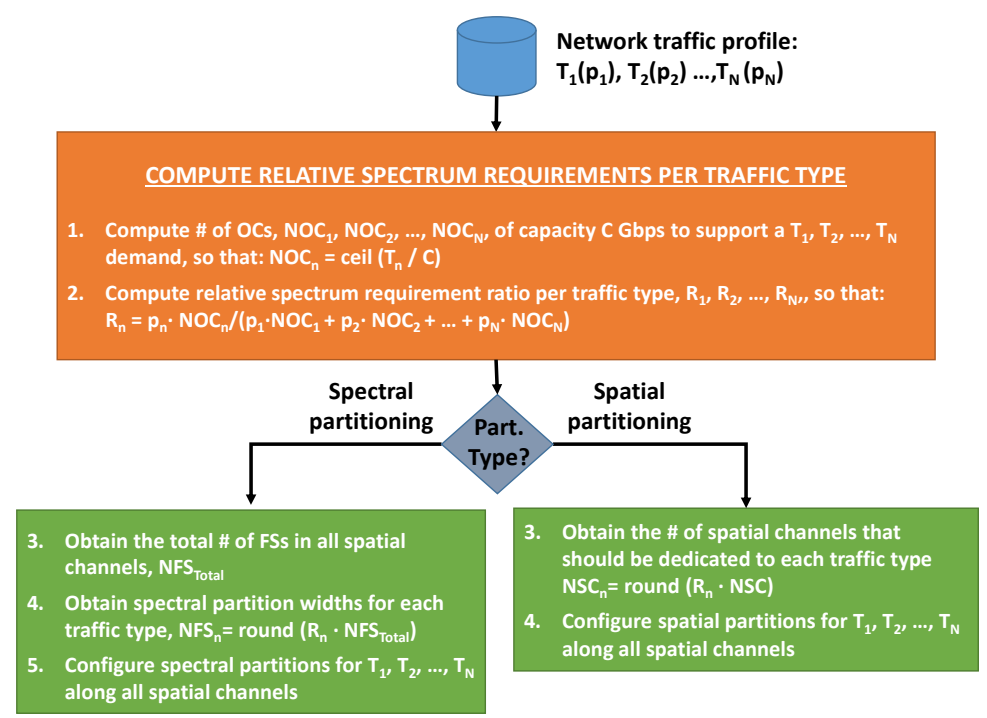

Figure 1. Flowchart describing spectral and spatial partitioning procedures 
The same relative spectrum requirements per traffic type could also be employed to perform an alternative yet simpler spatial partitioning, specifically designed for Flex-Grid/SDM networks, where full spatial channels are dedicated to each traffic type, as also depicted in the flowchart presented in Figure 1. In this case, the number of spatial channels dedicated to each traffic type, $N S C_{n}$, for all $n \in\{1, \ldots, N\}$, would have to be computed as $N S C_{n}=$ round $\left(R_{n} \cdot N S C\right)$, being $N S C$ the total number of available spatial channels and round() the same integer rounding function. Finally, spatial partitions of such sizes would be configured.

Imagine an example where the offered traffic profile is composed of $T_{1}=100 \mathrm{G}(40 \%), T_{2}=400 \mathrm{G}$ $(30 \%)$ and $T_{3}=1 \mathrm{~Tb} / \mathrm{s}(30 \%)$ connections. Moreover, imagine that the available optical modulators in the network offer a fixed baud-rate of $25 \mathrm{Gbaud}$, being Polarization Multiplexed (PM)-8-QAM employed in all cases, with a spectral efficiency equal to $6 \mathrm{bit} / \mathrm{s} / \mathrm{Hz}$. Under these assumptions, the effective bit-rate per $O C$ would be $C=150 \mathrm{~Gb} / \mathrm{s}$. Hence, $N O C_{1}=1, N O C_{2}=3$ and $\mathrm{NOC}_{3}=7$. This would lead to the following relative spectral requirements, eventually: $R_{1}=$ $11.75 \%, R_{2}=26.5 \%$ and $R_{3}=61.75 \%$.

Now, imagine a Flex-Grid/SDM network scenario equipping SMFBs of 7 independent SMFs per link, offering each one the $4 \mathrm{THz}$ C-Band, discretized into $320 \mathrm{FSs}$ of $12.5 \mathrm{GHz}$, for allocating connections. Therefore, NFS Total $=2240$ available along all spatial channels. If a spectral partitioning is performed in this scenario, $N F S_{1}=263, N F S_{2}=594$ and $N F S_{3}=1383$, thus resulting in the partitioning configuration depicted in Figure 2.a.

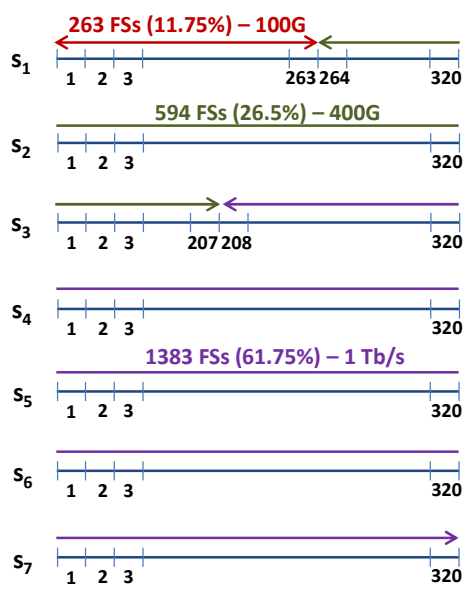

a)

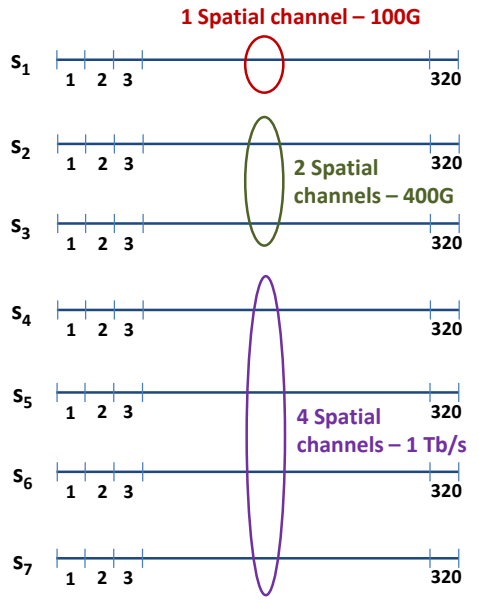

b)

Figure 2. Spectral (2.a) vs. spatial (2.b) partitioning example to allocate $100 \mathrm{G}, 400 \mathrm{G}$ and $1 \mathrm{~Tb} / \mathrm{s}$ connections in an SDM link with 7 independent SMFs. Offered traffic profile: $100 \mathrm{G}(40 \%), 400 \mathrm{G}(30 \%), 1 \mathrm{~Tb} / \mathrm{s}(30 \%)$.

Conversely, a spatial partitioning would organize the spatial partitions as depicted in Figure 2.b. Being NSC $=7$, the size of the different spatial partitions would be $N S C_{1}=0.82 \approx 1, N S C_{2}=1.86 \approx$ 2 and $N_{S C}=4.32 \approx 4$. It is obvious that in this case $1 \mathrm{~Tb} / \mathrm{s}$ connections are penalized, while more FS than the required are assigned to $100 \mathrm{G}$ and $400 \mathrm{G}$ connections. Nevertheless, this effect would be mitigated in scenarios where the number of spatial channels is increased and, therefore, the rounding effects are smaller. 
Either configuring spectral or a spatial partitioning as depicted in Figure 2, when a new 100G, $400 \mathrm{G}$ or $1 \mathrm{~Tb} / \mathrm{s}$ connection arrives at the network, the spectrum allowed to support it is limited to these partitions, selected in a first-fit fashion. Two advantages are foreseen when adopting these strategies vs. generic Flex-Grid: 1) Fragmentation is avoided as spectrum bands released after connections termination have the same size inside the different partitions; 2) The unfairness problem [19] typical of Flex-Grid scenarios, where large connections suffer higher blocking than smaller ones, is avoided, since partitions could be tailored to tune the network blocking performance. A potential drawback of both spectral/spatial partitioning can appear under unexpected traffic profile deviations, creating a mismatch between the offered traffic and the configured spectral/spatial partitions. When these traffic profile deviations become stable, network re-partitioning would be an option to allow the network continue delivering high performance. Lastly, comparing spectral and spatial partitioning, the latter seems simpler and easier to manage given its coarser granularity, which may facilitate connection allocation decisions in the network.

\section{NUMERICAL RESULTS}

An ad-hoc Java-based simulator has been implemented to obtain accurate numerical performance results of the previously described spectral and spatial partitioning strategies for Flex-Grid/SDM networks. In order to get results as generic as possible, different topologies as well as diverse traffic profiles have been simulated.

\subsection{Evaluation scenarios}

Two different topologies [12] have been considered for this study, namely, the DT12 German transport network (12 nodes, 20 links), whose diameter is $1019 \mathrm{~km}$, and the pan-European EON16 network (16 nodes, 23 links), with a diameter of $2663 \mathrm{~km}$. For the sake of simplicity, a single modulation format is employed in each network. According to [20], the maximum transmission reach of PM-8QAM is $1340 \mathrm{~km}$ in SDM networks deploying SMFB links, while this reach is increased to $3796 \mathrm{~km}$ when PM-QPSK is used. Therefore, when the DT12 is simulated, the maximum path length is limited to $1340 \mathrm{~km}$ and PM-8QAM is always used. In contrast, when simulating the EON16, the maximum path length increases to $3796 \mathrm{~km}$ and PM-QPSK is always employed.

We assume the available optical modulators in the network offer a fixed baud-rate of $25 \mathrm{Gbaud}$. So, if we introduce a guard-band between adjacent OCs equal to $1 \mathrm{FS}$, each OC ends occupying a total spectral width of $3 \mathrm{FSs}$, offering an effective transmission capacity of 150 or $100 \mathrm{~Gb} / \mathrm{s}$ when PM-8QAM (as in the DT12) or PM-QPSK (as in the EON16) is employed, with spectral efficiencies of 6 and $4 \mathrm{~b} / \mathrm{s} / \mathrm{Hz}$, respectively. As a result, to support 100G, $400 \mathrm{G}$ and $1 \mathrm{~Tb} / \mathrm{s}$ incoming demands in the DT12 network, super-channels composed of 1, 3 and 7 OCs will be needed, occupying 3, 9 and $21 \mathrm{FSs}$. In contrast, in the EON16 network, super-channels to support 100G, $400 \mathrm{G}$ and $1 \mathrm{~Tb} / \mathrm{s}$ incoming demands will need to be composed of 1,4 and $10 \mathrm{OCs}$, that is, occupying 3, 12 and $30 \mathrm{FSs}$. All connections will be supported over spectral super-channels in both networks. So, the aforementioned numbers of FSs will always need to be contiguously allocated in a specific spatial channel included in the allowed spectral/spatial partition. 
A SMFB is assumed to be deployed per network link in each direction, including a number of standard SMFs that can vary from 7 to 30 depending on the experiment. The $4 \mathrm{THz} \mathrm{C}-\mathrm{Band}$ is used in each SMF, which results in $320 \mathrm{FSs}$ of $12.5 \mathrm{GHz}$ width. In some cases, this number of FSs is slightly adjusted to better fit the spectral requirements of incoming connection requests. For example, with spatial partitioning, 324 FSs are enabled in those SMF dedicated to support 400G and $1 \mathrm{~Tb} / \mathrm{s}$ connections, still within the C-Band effectively. This number of FSs better matches the 9 or 12 FS required by 400 connections when PM-8-QAM or PM-QPSK is used. Otherwise, a significant amount of spectral resources would directly be wasted per SMF. In any case, to be totally fair, the same slightly modified number of FSs is equipped per SMF when testing spectral partitioning or no partitioning cases.

Regarding the Routing, Spatial channel and Spectrum Assignment (RSSA) algorithm, we employ a $K$-Shortest Path routing algorithm, constraining the candidate paths by the aforementioned maximum path length values. Once the $K$ candidate paths to support a given connection are obtained, these ones are explored starting from the shortest to the longest one. Note here that the specific number of paths to explore by the algorithm can be less than $K$ eventually, when some of the computed paths exceed the maximum transmission reach imposed by the employed modulation format. As for the spatial channel and spectrum assignment, a first-fit strategy is used, assuming that cost-effective InS without lane change SDM-ROADMs are present in the network. Hence, spatial channel continuity must be ensured end-to-end along the candidate paths, besides the spectrum continuity and contiguity constraints imposed by the Flex-Grid technology.

The worst-case time complexity of this RSSA algorithm can be expressed as follows. If $/ N /$ and $\mid E /$ denote the number of nodes and links in the network, the time complexity of computing the $K$ candidate paths using the well-known Yen's algorithm [21] is $O(K \cdot|N| \cdot(|E|+|N| \cdot \log (|N|))$. As for the spatial channel and spectrum assignment, the (very unlikely) worst-case would be given when there is only one spectral/spatial partition encompassing all spatial channels and the incoming demand requires a single $\mathrm{FS}$, needing up to $O\left(K \cdot F S_{\text {Total }}\right)$ continuous $F S$ availability checks along the $K$ candidate paths (recall that $F S_{\text {Total }}$ denotes the total number of available FSs along all spatial channels). Therefore, the worst-case time complexity of the RSSA algorithm becomes $\mathrm{O}\left(K \cdot|N| \cdot\left(|E|+|N| \cdot \log (|N|)+K \cdot F S_{\text {Total }}\right)\right.$ eventually.

In our simulations, two different traffic profiles have been considered, whose parameters are summarized in Table 1, where ABRC and AFSC refer to Average Bit-Rate per Connection and Average number of FS per connection, respectively.

Table1. Offered traffic profiles

\begin{tabular}{|c|c|c|c|c|c|c|c|c|c|c|c|c|}
\hline \multirow[t]{2}{*}{ Traffic Profile } & \multicolumn{3}{|c|}{ Connections Ratio (\%) } & \multicolumn{3}{|c|}{$\begin{array}{c}\text { Spectrum Used DT12 } \\
\text { (\%) }\end{array}$} & \multicolumn{3}{|c|}{$\begin{array}{c}\text { Spectrum Used EON16 } \\
(\%)\end{array}$} & \multirow{2}{*}{$\begin{array}{l}\text { ABRC } \\
(\mathrm{Gb} / \mathrm{s})\end{array}$} & \multirow{2}{*}{$\begin{array}{c}\text { AFSC } \\
\text { DT12 } \\
\text { (FS) }\end{array}$} & \multirow{2}{*}{$\begin{array}{c}\text { AFSC } \\
\text { EON16 } \\
\text { (FS) }\end{array}$} \\
\hline & 100G & 400G & $1 \mathrm{~Tb} / \mathrm{s}$ & 100 & 400 & $1 \mathrm{~Tb} / \mathrm{s}$ & 100 & 400 & $1 \mathrm{~Tb} / \mathrm{s}$ & & & \\
\hline TP1 (Current) & 70 & 20 & 10 & 35 & 30 & 35 & 28 & 32 & 40 & 250 & 6 & 7.5 \\
\hline TP2 (Future) & 40 & 30 & 30 & 11.765 & 26.47 & 61.765 & 8.7 & 26.1 & 65.2 & 460 & 10.2 & 13.8 \\
\hline
\end{tabular}

It is important to highlight that the fraction of spectrum expected to be required per connection type differs from their contribution to the total offered traffic in the DT12 network, but not in the EON16 one. For example, under TP1, 100G, 400G and $1 \mathrm{~Tb} / \mathrm{s}$ connections will contribute to 
$28 \%, 32 \%$ and $40 \%$ of the total offered traffic, which matches their relative spectrum requirements in the EON16. This is due to the fact the ratio of FSs needed $(3,12$ and $30 \mathrm{FSs})$ vs. connection bit-rate (100G, 400G, $1 \mathrm{~Tb} / \mathrm{s}$ ) keeps constant when PM-QPSK is employed in the EON16. However, this does not happen when employing PM-8QAM in the DT12.

As explained before, one of the objectives of this work is to gauge the effect of increasing the number of spatial channels on the results obtained. Therefore, different values have been simulated. Specifically, 5 illustrative SDM spatial multiplicities, namely, 7, 12, 19, 22 and 30, have been evaluated, assuming SMFBs per link comprising such numbers of parallel SMFs.

\subsection{Numerical results and comparison}

First simulations have focused on the effects of different resource partitioning strategies. Three different cases are shown in Figure 3, where TP1 (see Table 1) has been offered:

- NOP (No partitioning): The connection demands are allocated without any distinction, using all the spatial channels and occupying them from low to high spatial channels in a first-fit spatial channel and spectrum assignment.

- SPAP (Spatial partitioning): Each type of connections uses its configured spatial partition, as described in Figure 2.b. In the case shown in Figure 3, with 7 spatial channels, 100G connections are allocated over the two first spatial channels, 400G ones over spatial channels 3 and 4 , and $1 \mathrm{~Tb} / \mathrm{s}$ connections can use channels from 5 to 7 .

- SPECP (Spectral Partitioning): Each type of connections use its configured spectral partition, as described in Figure 2.a.

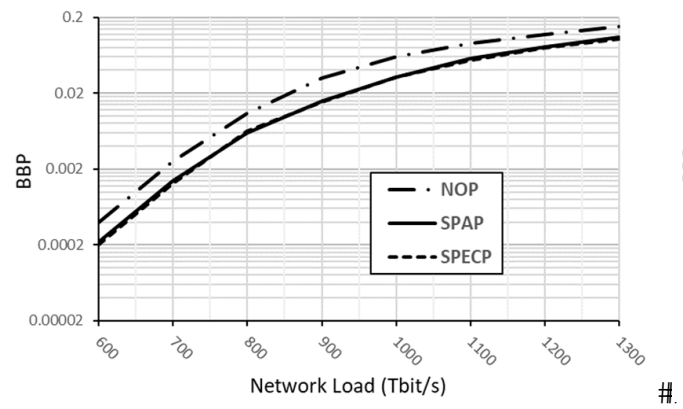

(a)

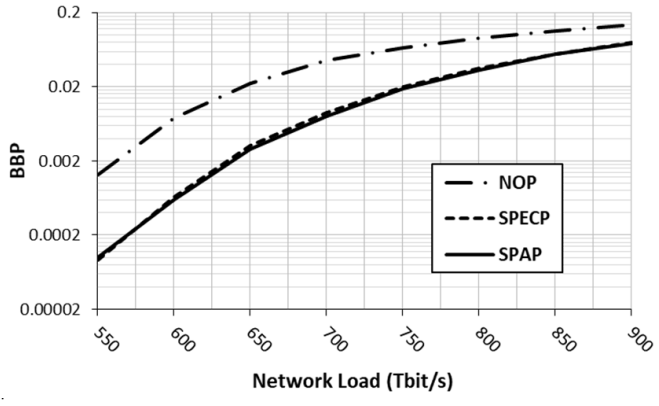

(b)

Figure 3. Bandwidth blocking probability vs. average supported network load in the DT12 (a) and EON16 (b) networks when TP1 is generated.

Some conclusions can be extracted from Figure 3. First and foremost, both SPAP and SPECP are always beneficial in terms of supported load at any BBP value versus NOP, providing SPECP slightly better performance. Specifically, it is observed that gains in the EON16 network are clearly higher than those obtained in the DT12, where the performance of all options is similar. This happens as a result of the higher differences between larger and smaller connections sizes when employing PM-QPSK in the EON16. Looking at the load values for $B B P=1 \%$, these are 795 , 855 and 860 Tbit/s for NOP, SPAP and SPECP in the DT12, respectively (as seen in Figure 3.a, differences between SPAP and SPECP are very small). In contrast, in the EON16 (Figure 3.b) the relative differences between the NOP case and those using partitions (SPAP and SPECP) is clearly higher, namely, 610, 705 and $710 \mathrm{Tbit} / \mathrm{s}$ at $\mathrm{BBP}=1 \%$. While load gains are around $8 \%$ in the 
DT12, they rise to about $16 \%$ in the EON16.

A similar behavior is observed when TP2 is generated, but in this case differences between SPAP and SPECP are higher (see Figure 4). This is due to the fact that, by having higher differences in the proportions of spectrum dedicated to each kind of connections (see Table 1), the differences between assigning complete fibers or the exact number of slots become larger.

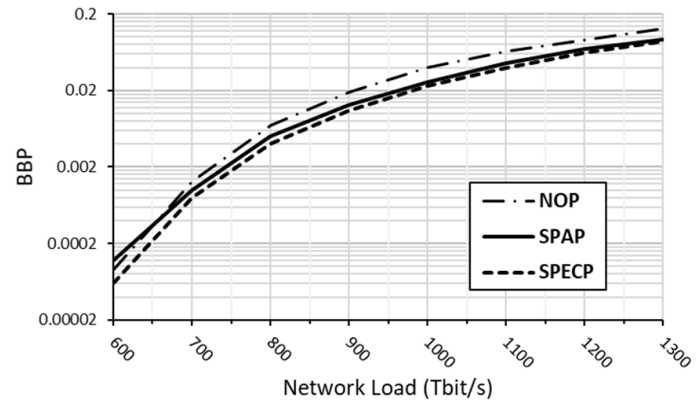

(a)

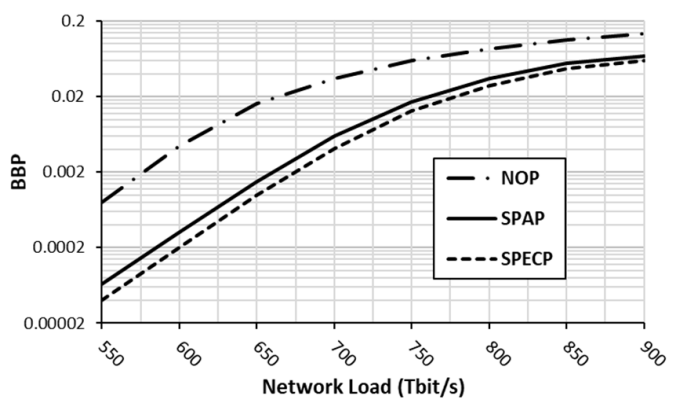

(b)

Figure 4. Bandwidth blocking probability vs. average supported network load in the DT12 (a) and EON16 (b) networks when TP2 is generated.

Therefore, from Figure 4 it can be concluded that the differences between the SPAP and SPECP strategies are larger under TP2. Looking at the exact load values for BBP $=1 \%$, these are 845,875 and $900 \mathrm{Tbit} / \mathrm{s}$ for NOP, SPAP and SPECP in DT12, respectively (Figure 4.a). In contrast, in the EON16 (Figure 4.b) the relative differences are clearly higher, namely, 630, 730 and $740 \mathrm{Tbit} / \mathrm{s}$ at $\mathrm{BBP}=1 \%$. While load gain values obtained by spectrum partitioning range from $3 \%$ to $6 \%$ in the DT12, they rise to a range from $15 \%$ to almost $18 \%$ in the EON16.

As mentioned before, one potential drawback of spectral/spatial partitioning could stem from a mismatching between the partitions and the offered traffic profile. With the aim of quantifying this effect, some additional simulations have been also conducted where the offered load deviates from the expected one, but without modifying the configured partitions. The obtained results are summarized in Figure 5, considering the EON network under TP1 and BBP $=5 \%$, where SPAP and NOP have been compared. Please recall from Figure 3.b that negligible performance differences between SPAP and SPECP exist in the EON under TP1. Therefore, very similar results with SPECP would also be observed.

The traffic profile deviations simulated consist in decreasing the ratio of $100 \mathrm{G}$ connections (whose value in TP1 is 70\%) in steps of 5\%, increasing that of $400 \mathrm{G}$ and $1 \mathrm{~Tb} / \mathrm{s}$ connections by $2.5 \%$ each. For example, the first deviated traffic profile becomes 100G (65\%), $400 \mathrm{G}(22.5 \%)$ and $1 \mathrm{~Tb} / \mathrm{s}$ (12.5\%). 


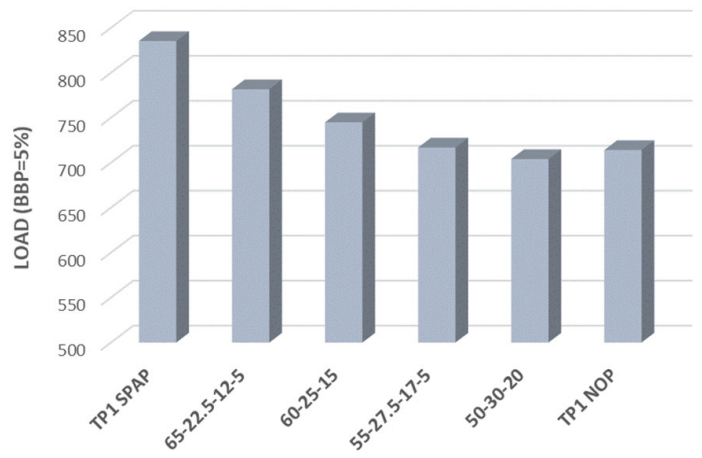

Figure 5. Supported load at $\mathrm{BBP}=5 \%$ when there is a mismatch between traffic profile and spectrum partitioning. Current traffic (TP1) and EON network are considered.

Extreme left and right bars in Figure 5 gather the results obtained when SPAP (left bar) and NOP (right bar) cases are simulated. Under the deviated traffic profiles (whose percentages are given in $\mathrm{x}$-axis) it is seen how the gain obtained by using SPAP gradually decreases, becoming almost zero when the $100 \mathrm{G}-400 \mathrm{G}-1 \mathrm{~Tb} / \mathrm{s}$ offered traffic is $55 \%-27.5 \%-17.5 \%$. From this point, NOP becomes better than using SPAP. Nevertheless, when deviations from the expected profile are small, partitioning is still worth. For example, when the offered traffic is $60 \%-25 \%-15 \%$, the load supported using SPAP is still $4 \%$ higher than that supported with NOP.

A second set of simulations was devoted to study the effect of an increased spatial multiplicity in the gain obtained by SPAP and SPECP. Indeed, it seems plausible that the larger the number of spatial channels, the smaller the differences between them (i.e., differences between assigning whole fibers versus assigning the number of slots proportional to the fraction of each type of connections in the total load). Results obtained are shown in Figure 6.

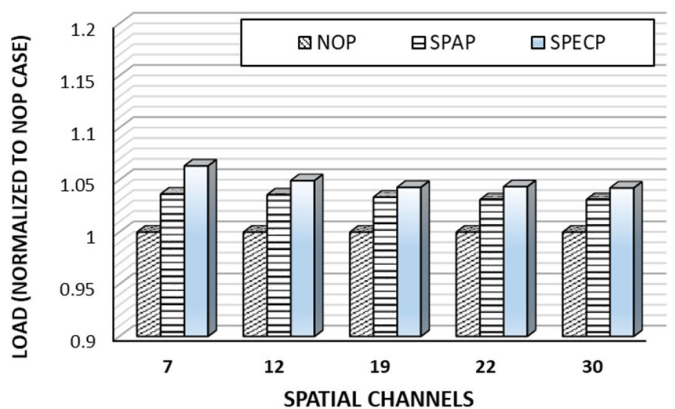

(a)

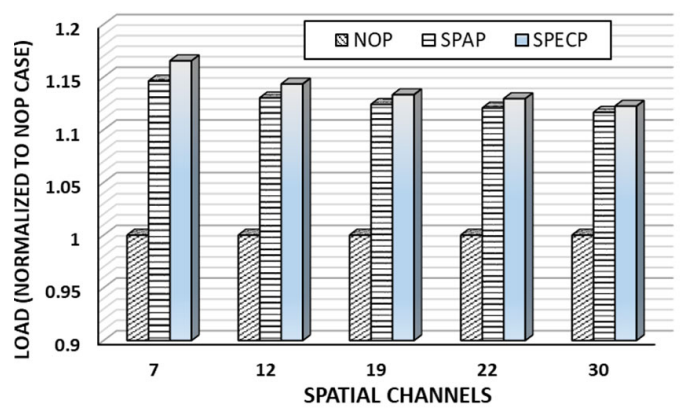

(b)

Figure 6. Supported load for SPAP and SPECP compared to NOP (Load=1 for this later case) in the DT12 (a) and EON16 (b) networks, keeping BBP $=1 \%$. Spatial multiplicities equal to 7, 12, 19, 22 and 30 are evaluated.

As expected, it can be observed that, independently of the network topology, the gain obtained by SPAP and SPEC versus NOP is maximal when the number of parallel spatial channels takes its minimum value ( 7 in our study). The best performance is always obtained by SPECP, but its advantage over SPAP reduces when increasing the spatial multiplicity.

For example, Figure 6.a shows that SPAP gain versus NOP in the DT12 network stabilizes around 
$3.1 \%$ when the number of spatial channels is large, while SPECP tends to a value of $4.2 \%$. Taking into account that the load supported by this network is $4024 \mathrm{Tbit} / \mathrm{s}$ when using NOP, these extra $3.1 \%$ and $4.2 \%$ correspond to an increase of $125 \mathrm{~Tb} / \mathrm{s}$ and $170 \mathrm{~Tb} / \mathrm{s}$ in the average network load when SPAP and SPECP are used, respectively.

Similar trends are observed in the EON16 (Figure 6.b). In this case, SPECP gain reduces slowly from almost $16 \%$ with 7 spatial channels to around $12 \%$ when the number of spatial channels rises up to 30 . SPAP gains also reduce from $14.5 \%$ to around $11.5 \%$ when increasing the spatial multiplicity from 7 to 30 . In this case, a gain in supported network load ranging from 11 to $12 \%$ is observed, meaning an extra load between 360 and $390 \mathrm{~Tb} / \mathrm{s}$ (network load at BBP=1\% and 30 spatial channels correspond in this case to $3165 \mathrm{~Tb} / \mathrm{s})$.

Summarizing the results obtained during simulations, SPECP is always the best case, but its advantage over SPAP becomes almost negligible when increasing the number of spatial channels, regardless of the considered topology. Extra network load supported by applying spectrum partition mechanisms ranges from $5 \%$ to $17 \%$ depending on the different network topologies and conditions.

\section{CONCLUSIONS AND FUTURE WORK}

In this work, we analyze the effects of partitioning the spectral resources in Flex-Grid/SDM networks. Taking into account that spectrum fragmentation limits the performance of this kind of networks, we propose the isolated assignment of the different connections types by partitioning the available resources. Two different strategies have been compared: SPECP, where the spectral resources assigned to each kind of connections corresponds to the expected spectrum consumed according to the generated traffic profile; SPAP, where an integer number of spatial channels is assigned to each kind of connection. It has been assessed how, in some scenarios, SPAP provides results very close to those obtained by applying SPECP which is considered the best case. When the number of spatial channels is high and/or the percentages of the different connection types are similar, the differences between both strategies reduce and performance attained by using SPAP is very close to that of SPECP. Therefore, given the higher simplicity of SPAP vs. SPECP, the obtained results make us advocate for SPAP as a more interesting solution for spectrum fragmentation avoidance in Flex-Grid/SDM optical networks.

As a future work, we plan to investigate new SDM-ROADM architectures applicable to spatiallypartitioned Flex-Grid/SDM optical network scenarios yielding superior hardware complexity \& cost reduction, even higher than existing JoS-based or InS without lane-change SDM-ROADM ones.

\section{ACKNOWLEDGEMENTS}

This work has been partially funded by Spanish projects TWINS (TEC2017-90097-R) and ALLIANCE (TEC2017-90034-C2-1-R).

\section{REFERENCES}

[1]. M. Jinno, H. Takara, B. Kozicki, Y. Tsukishima, Y. Sone, S. Matsuoka, "Spectrum-Efficient and Scalable Elastic Optical Path Network: Architecture, Benefits, and Enabling 
Technologies," IEEE Commun. Mag., vol. 47, no. 11, Nov. 2009.

[2]. O. Gerstel, M. Jinno, A. Lord, S. J. Ben Yoo, "Elastic optical networking: A new dawn for the optical layer?," IEEE Commun. Mag., vol. 50, no. 2, pp. 12-20, Feb. 2012.

[3]. M. Ruiz, L. Velasco, A. Lord, D. Fonseca, M. Pioro, R. Wessaly, J. P. Fernandez-Palacios, "Planning Fixed to Flexgrid Gradual Migration: Drivers and Open Issues," IEEE Commun. Mag., vol. 52, no. 1, pp. 70-76, Jan. 2014.

[4]. International Telecommunication Union - ITU-T, "G.694.1 (02/2012), Spectral grids for WDM applications: DWDM frequency grid," Ser. G.694.1, pp. 1-16, 2012.

[5]. R.-J. Essiambre, G. Kramer, P. J. Winzer, G. J. Foschini, B. Goebel, "Capacity Limits of Optical Fiber Networks, " IEEE/OSA J. Lightwave Technol., vol. 28, no. 4, pp. 662-701, Feb. 2010.

[6]. P. J. Winzer, "Spatial Multiplexing in Fiber Optics: The 10x Scaling of Metro/Core Capacities," Bell Labs Tech. J., vol. 19, pp. 22-30, 2014.

[7]. D. J. Richardson, J. M. Fini, L. E. Nelson, "Space Division Multiplexing in Optical Fibres," Nat. Photonics, vol. 7, pp. 354-362, 2013.

[8]. D. Klonidis, F. Cugini, O. Gerstel, M. Jinno, V. Lopez, E. Palkopoulou, M. Sekiya, D. Siracusa, G. Thouénon, C. Betoule, "Spectrally and spatially flexible optical network planning and operations," IEEE Commun. Mag., vol. 53, no. 2, pp. 69-78, 2015.

[9]. L. E. Nelson, M. D. Feuer, K. Abedin, X. Zhou, T. F. Taunay, J. M. Fini, B. Zhu, R. Isaac, R. Harel, G. Cohen, D. M. Marom, "Spatial superchannel routing in a two-span ROADM system for space division multiplexing," J. Light. Technol., vol. 32, no. 4, pp. 783-789, 2014.

[10].B. Shariati, J. M. Rivas-Moscoso, D. M. Marom, S. Ben-Ezra, D. Klonidis, L. Velasco, I. Tomkos, "Impact of spatial and spectral granularity on the performance of SDM networks based on spatial superchannel switching," J. Light. Technol., vol. 35, no. 13, pp. 2559-2568, 2017.

[11].D. M. Marom, P. D. Colbourne, A. D’Errico, N. K. Fontaine, Y. Ikuma, R. Proietti, L. Zong, J. M. Rivas-Moscoso, I. Tomkos, "Survey of Photonic Switching Architectures and Technologies in Support of Spatially and Spectrally Flexible Optical Networking," J. Opt. Commun. Netw., vol. 9, no. 1, pp. 1-26, Jan. 2017.

[12].R. Rumipamba-Zambrano, F.-J. Moreno-Muro, J. Perelló, P. Pavón-Mariño, S. Spadaro, "Space Continuity Constraint in Dynamic Flex-Grid/SDM Optical Core Networks: An Evaluation with Spatial and Spectral Super-channels," Comput. Commun., vol. 126, pp. 3849, Aug. 2018.

[13].B. C. Catterjee, N. Sarma, E. Oki, "Routing and Spectrum Allocation in Elastic Optical Networks: A Tutorial," IEEE Commun. Surv. Tut., vol. 17, no. 3, pp. 1776-1800, third quarter 2015.

[14].J. Yuan, R. Zhu, Y. Zhao, Q. Zhang, X. Li, D. Zhang, A. Samuel, "A Spectrum Assignment Algorithm in Elastic Optical Network with Minimum Sum of Weighted Resource Reductions in all Associated Paths," J. Light. Technol., vol. 37, no. 21, pp. 5583-5592, Nov. 2019.

[15].J. Yuan, Z. Xu, R. Zhu, Q, Zhang, X. Li, J. Zhang, "A pre-split multi-flow RMSA algorithm in elastic optical networks", Optical Fiber Technology, vol. 52, Nov. 2019.

[16].Y. Zhao, L. Hu, R. Zhu, X. Yu, Y. Li, W. Wang, J. Zhang, "Crosstalk-aware spectrum defragmentation by re-provisioning advance reservation requests in space division multiplexing enabled elastic optical networks with multi-core fiber," Opt. Express, vol. 27, no. 4, pp. 5014-5032, Feb. 2019.

[17].H. Yang, Q. Yao, A. Yu, Y. Lee, J. Zhang, "Resource Assignment Based on Dynamic Fuzzy 
Clustering in Elastic Optical Networks with Multi-Core Fibers," in IEEE Transactions on Communications, vol. 67, no. 5, pp. 3457-3469, May 2019.

[18].Y. Qiu, "Group-based spectrum assignment in dynamic flex-grid optical networks," Optical Fiber Technology, vol. 19, no. 5, pp. 437-445, Oct. 2013.

[19].R. Wang, B. Mukherjee, "Spectrum management in heterogeneous bandwidth optical networks," Optical Switch. and Netw., vol. 11, pp. 83-91, Jan. 2014.

[20].B. Shariati, A. Mastropaolo, N.-P. Diamantopoulos, J. M. Rivas-Moscoso, D. Klonidis, I. Tomkos, "Physical-layer-aware performance evaluation of SDM networks based on SMF bundles, MCFs, and FMFs, " J. Opt. Commun. Netw., vol. 10, no. 9, pp. 712-722, Sep. 2018.

[21].J. Y. Yen, "Finding the K shortest loopless paths in a network," Manage. Sci., vol. 17, no. 11, Jul. 1971 